\title{
Emotional arousal ripples across time to bind subsequent episodes in memory
}

\author{
David Clewett ${ }^{1} \&$ Lila Davachi ${ }^{2,3^{*}}$ \\ 1Department of Psychology, University of California, Los Angeles USA \\ ${ }^{2}$ Department of Psychology, Columbia University, USA \\ ${ }^{3}$ Nathan Kline Institute, Orangeburg, New York, USA
}

*To whom correspondence should be addressed:

Department of Psychology

Columbia University

Schermerhorn Hall \#406

1190 Amsterdam Ave

New York, NY 10027

Email: Id24@columbia.edu

Keywords: episodic memory, arousal, emotion, temporal, sequence, event boundary, event segmentation

Competing Interests: The authors declare no competing interests.

Acknowledgements: This project was funded by federal NIH grant R01 MH074692 to L.D. and by federal NIH grant F32 MH114536 to D.C. The authors thank Zala Reppman and Natalie Plotkin for their assistance with data collection. We also thank Dr. Alexandra Cohen, Dr. Elizabeth Goldfarb, Camille Gasser, and Mason McClay for their helpful feedback on earlier versions of this manuscript. 


\section{Abstract}

Time unfolds continuously, yet our memories are stored as discrete episodes. Prior work shows that fluctuations between stability and change in an ongoing neutral context facilitates this formation of distinct and memorable events. However, less is known about how shifting emotional states influence these memory processes, despite ample evidence that emotion has a robust influence on non-temporal aspects of episodic memory. Here, we examined if emotional stimuli influence temporal memory for recent event sequences. Participants encoded lists of neutral object images while listening to pure auditory tones. At regular intervals within each list, participants heard emotional positive, negative, or neutral sounds, which served as 'emotional event boundaries' that divided each sequence into discrete auditory events. Temporal order memory was tested for neutral item pairs that either spanned an emotional sound ('boundary-spanning') or encountered within the same auditory event ('same-context'). We found that highly arousing boundaries had opposite effects on binding ongoing versus subsequent sequential representations in memory. Specifically, highly arousing emotional sounds tended to lead to worse temporal order memory for boundary-spanning item pairs. By contrast, they led to better temporal order memory for same-context item pairs in the next event. Both of these arousal effects were specific to negative sounds. The carryover effect of negative arousal was also strongest for item pairs encountered closest to the boundary and diminished as the event unfolded. These findings suggest that temporally dynamic emotional states support the temporal integration of mnemonic events, which may contribute to the hyper-episodic nature of negative emotional memories. 


\section{Introduction}

Our lives unfold as continuous narratives. Yet like chapters in a book, our memories of such experiences usually take the form of discrete and meaningful episodes. A rapidly growing body of work demonstrates that such memory organization may be driven by a dynamic interplay between temporal stability and change in different contextual features across time (Brunec et al., 2018; Clewett \& Davachi, 2017; Clewett et al., 2019). For example, it has been shown that following list learning, temporal order memory is impaired for item pairs that spanned a change in background color (Heusser et al., 2018), spatial location (Horner et al., 2016), perceptual category (DuBrow \& Davachi, 2013, 2014), or accompanying sounds (Clewett et al., 2020) compared to item pairs that shared similar contextual information. Thus, while contiguities in one's surrounding context may facilitate the binding of sequential representations in episodic memory, event boundaries (i.e., context shifts) seem to disrupt this process and lead to the formation of discrete mnemonic events.

Much of the research on event cognition to date has focused on how simple changes in the external environment, such as a change in location or local perceptual features, affect the organization of episodic memory (Clewett et al., 2019). However, everyday experiences are also colored by shifting emotional states and fluctuations in arousal. Emotional stimuli elicit robust effects on perception, attention, and memory (Kensinger et al., 2007; LaBar \& Cabeza, 2006; Mather \& Sutherland, 2011; McGaugh, 2013). Yet, somewhat surprisingly, little work has examined how emotional arousal and valence influence the temporal aspects of episodic memory, even though this rich sequential information is a defining feature of everyday memory (Palombo \& Cocquyt, 2020). The few studies that have investigated the effects of emotion on temporal memory have also yielded inconsistent findings, whereby emotion can sometimes enhance (Knight \& Mather, 2009; Palombo et al., 2021; Rimmele et al., 2012; Schmidt et al., 2011) and other times impair memory for the precise timing of individual items (D'Argembeau \& Van der Linden, 2005) or the temporal order of emotionrelated events (Huntjens et al., 2015). Likewise, emotion has been shown to disrupt temporal contiguity effects in free recall, with the output order of retrieval instead reflecting greater semantic clustering between items with negative valence (Long et al., 2015; Talmi et al., 2019). This suggests that emotion might in and of itself serve as a strong internal context for linking or separating memories across time.

While it is clear emotion has complex effects on memory for both the timing and temporal order of stimuli, current findings do not address how emotion shapes the episodic structure of memory, per se, especially for neutral details encountered in those contexts. For decades, the memory literature has been dominated by list-based paradigms that randomize and/or fully block the presentation of emotional and neutral items (Barnacle et al., 2018; D'Argembeau \& Van der Linden, 2005; Long et al., 2015; Talmi et al., 2019). But this procedure results in sequences that lack the temporal stability and change in ongoing contextual features that seem to be necessary for constructing distinct episodic memories (Clewett et al., 2019; Siefke et al., 2019). Another important limitation is that prior work often uses aversive stimuli that elicit sustained increases in arousal or can alter an individual's mood. For instance, it has been shown that temporally extended emotional contexts, such as aversive videos or exposure to physiological stressors, affect participants' abilities to remember when a recent event occurred (Zlomuzica et al., 2016) and to learn sequential associations 
(Maran et al., 2017). Prolonged elicitations of arousal and stress, however, likely obscure any underlying effects of emotion on discrete event segmentation processes. Without discrete manipulations of arousal, it is challenging to determine if shifting emotional states also play an important role in structuring episodic memories.

Understanding the consequences of emotion on temporal memory organization is especially important given recent evidence that even the simplest context shifts elicit increased pupil dilation, an index of physiological arousal (Clewett et al., 2020; Kahneman \& Beatty, 1966). In one pupillometry study, these surges in arousal were also linked to corresponding impairments in temporal order memory, a common index of event parsing in episodic memory (Clewett et al., 2020). This finding raises the intriguing possibility that emotional stimuli may function as especially strong event boundaries by eliciting robust increases in arousal-related event segmentation. Supporting this idea, emotionally arousing stimuli exert strikingly similar effects on memory and attention as do event boundaries, including enhancing item recognition (Cahill \& McGaugh, 1998; Dolan, 2002; Swallow et al., 2009), increasing attentional allocation (Bradley et al., 1997; Mather \& Sutherland, 2011; Zacks et al., 2007), and enhancing item-source memory for concurrent contextual information (Clewett et al., 2020; Heusser et al., 2018; Kensinger et al., 2006; Rimmele et al., 2012). Recent work also shows that prediction errors, which are associated with an increase in autonomic arousal (Preuschoff et al., 2011; Raisig et al., 2010), lead to impairments in temporal order memory (Rouhani et al., 2019). Both emotional stimuli and event boundaries also elicit subjective distortions in perception and memory for the duration of recent events (Brunec et al., 2017; Droit-Volet \& Gil, 2009; Ezzyat \& Davachi, 2014; Faber \& Gennari, 2015; Johnson \& MacKay, 2019; Lake et al., 2016; Liverence \& Scholl, 2012), supporting the notion that arousal mechanisms are sensitive to salient changes in the world and mediate the impact of contextual shifts on long-term memory.

While an emotional stimulus, such as a gruesome image, may be encountered only briefly, its effects on cognition do not occur in isolation. Rather, the effects of emotional items can spillover in time to influence the memorability of temporally-adjacent details. A large body of work shows that embedding emotionally arousing oddball items within more mundane item sequences can enhance or impair item recognition for both preceding and ensuing neutral items (Anderson et al., 2006; Clewett et al., 2017; Hurlemann et al., 2005; Knight \& Mather, 2009; Sakaki et al., 2014; Schluter et al., 2019; Strange et al., 2003). The direction of these modulatory effects of emotional arousal on memory also seems to depend, at least in part, on the temporal proximity of nearby neutral items (Bocanegra \& Zeelenberg, 2009; Schmidt \& Schmidt, 2016). While these findings suggest that arousal effects may linger in time to influence the memorability of individual items, it is unknown whether and how this spillover relates to temporal encoding processes.

Alongside more general arousal effects, it is important to consider that emotional valence may also play a role in modulating the temporal structure of memory. Emotional valence has been shown to elicit opposing effects on episodic memory (Kensinger, 2004; Pierce \& Kensinger, 2011). While negatively valenced stimuli tend to lead to more item-focused processing and memory, positive emotional stimuli are more likely to enhance associative memory (Clewett \& Murty, 2019; Kensinger, 2009; Madan et al., 2019). Positive and negative emotions have also been shown to exert distinct effects on attention, with positive valence broadening the scope 
of cognitive processing and negative valence narrowing the scope of attention (Gable \& Harmon-Jones, 2008, 2010). In parallel, it has been shown that orienting individuals to be more item-focused while encoding neutral item sequences can lead to impairments in subsequent serial recall (DuBrow \& Davachi, 2013). These converging findings suggest that by recruiting greater item-focused attention, negatively valenced stimuli may be more likely to elicit the most robust impairments in temporal order memory between information that spans them.

The goal of the current study was to examine how emotional arousal and valence influence the temporal organization of events in memory. To this end, we adapted an existing auditory event boundary paradigm by inserting emotional sounds into a sequence of neutral object images, and then examined how these 'emotional event boundaries' influenced temporal order memory for item pairs spanning those boundaries (discrete effect) as well as for item pairs appearing in the following event (carryover effect). We predicted that emotional sounds rated as being more arousing would correlate with worse temporal order memory accuracy for neutral item pairs spanning those sounds, consistent with emotion functioning as a strong event boundary during continuous experience. In contrast, we hypothesized that the effects of highly arousing emotional sounds would carry forward in time to enhance rather than impair temporal memory binding between item pairs in the ensuing auditory event. Such benefits would be consistent with the idea that emotion tags the ensuing context as motivationally relevant, making an otherwise mundane series of neutral stimuli more memorable. With respect to valence, we predicted that emotional sounds that are perceived as being more negative would correlate with greater temporal order memory impairments more broadly, given prior evidence that negative emotional stimuli tend to enhance item-focused processing at the expense of associative memory (Bisby \& Burgess, 2014).

\section{Methods}

Participants. Twenty-three healthy young adults were recruited from nearby community in New York City to participate in this experiment through advertisements on RecruitMe (https://recruit.cumc.columbia.edu). Two participants were excluded due to failure to follow instructions, and one participant was excluded due to a programming error that led to one experiment block being repeated. This left a total of twenty participants for data analysis $\left(M_{\text {age }}=21.8, \mathrm{SD}_{\text {age }}=4.65 ; 18 \mathrm{~F}\right.$ ). All participants provided written informed consent approved by the Columbia University Institutional Review Board and received monetary compensation. All eligible individuals had normal or normal-to-corrected vision and hearing and were not taking beta-blockers or psychoactive drugs.

Prior to starting the experiment, we performed a power analysis in $G^{*}$ Power 3.1 to estimate the appropriate sample size. The power analysis was carried out using data from three similar event boundary experiments (Clewett et al., 2020). In those studies, auditory event boundaries were shown to modulate two aspects of temporal memory: temporal order memory and temporal distance memory. The power analyses with an alpha $=.05$ and power $=.80$ (pooled from the average Cohen's $\mathrm{d}$ values from the three experiments) indicated we needed 40 participants to obtain a large effect size $(d=.80$; Cohen's criteria) to capture the 
weakest memory effect, temporal distance ratings However, a separate power analysis with the same parameters on the stronger event boundary memory effect, temporal order memory, indicated that only 19 participants were needed to obtain a large effect size (alpha $=.05$ and power $=.80, d=.80$; Cohen's criteria). Due to the COVID-19 pandemic, data collection was disrupted and we were only able to enroll 23 participants. Accordingly, we only report the results from the temporal order memory test, which had sufficient statistical power for analysis.

Materials. A total of 384 images of everyday neutral objects were selected from previous datasets (Gabrieli et al., 1997; Kensinger et al., 2006). The luminance of all object images and fixation screens was normalized using the SHINE toolbox in MATLAB. To manipulate the auditory context during the item sequences, three 1-s pure tones with sine waveforms of different frequencies $(600 \mathrm{~Hz}, 700 \mathrm{~Hz}, 800 \mathrm{~Hz}$, ) were generated using Audacity (https://www.audacityteam.org/). These frequencies were chosen such that sounds were discriminable from one another and were salient enough to maintain participants' attention. A total of 20 positive (e.g., laughing, pleasant music), 20 negative (e.g., screaming, gunshots), and 24 neutral (e.g., clock ticking, ambient mumbling) stimuli were selected from the International Affective Digitized Sound system (IADS) database (Bradley \& Lang, 2007) and the Internet. All auditory stimuli were edited using Audacity to be two seconds long in order to capture the most emotionally salient part of each clip. The volume of the audioclips was normalized using The Levelator (http://www.conversationsnetwork.org/levelator).

Sequence encoding. To examine how emotion influences the temporal binding of nearby neutral items, we adapted an existing event sequence task (Clewett et al., 2020). Participants viewed different sequences of 24 object images (Figure 1). Each image was displayed for $2.5 \mathrm{~s}$ followed by a 3 -s central fixation cross on a gray background. To create a stable auditory context, or event, a 1-s pure tone with a frequency of 600,700 or $800 \mathrm{~Hz}$ was played in the participant's left or right ear starting $0.5-\mathrm{s}$ into each fixation period. The side of the tone cued which hand should be used to judge whether the next object was larger than a shoebox (e.g., left ear = left hand). The ear/hand remained the same for 8 sequential objects. This ear laterality manipulation was used to ensure that participants perceived a series of contextually-distinct events, while the variety of withinevent pure tones was used to help maintain participants' attention across the task. The same pure tone was repeated within a given list.

After participants saw the $8^{\text {th }}$ object image in each auditory event, a trial-unique 2-s naturalistic audioclip was played in the opposing ear, thereby creating a salient auditory context shift, or 'event boundary'. This served as the first of two event boundaries within a list. Each naturalistic audio-clip was emotionally positive (pleasant), negative (unpleasant), or neutral and varied in its level of arousal. After hearing a naturalistic audioclip, participants viewed the next 8 object images, each preceded by the same neutral pure tone specific to that list (e.g., $800 \mathrm{~Hz}$ ). After the $8^{\text {th }}$ item in this second auditory event, a new naturalistic audio-clip was played in the opposite ear. This served as the second event boundary within a list. This event boundary audio-clip was then followed by the same pure tone being played in that same ear for the remainder of the items in the list. In 
total, each list contained two naturalistic audio-clips, or event boundaries, which served to parse each 24-item sequence into 3 discrete auditory events with 8 object images each. The type (i.e., pitch) of each pure tone used as same-context sounds was randomized across lists, and the ear that the tones first played in (left or right) was counterbalanced across lists. Participants viewed a total of 16 lists/sequences.

To reduce potential interactions between sound valences and boundary positions within a list $\left(1^{\text {st }}\right.$ or $2^{\text {nd }}$ ), we constructed two types of lists: mixed-valence lists (one emotional and one neutral) and pure-valence lists (only negative, neutral or positive). In the mixed lists, a positive or negative sound served as either the first or second event boundary, while the other event boundary sound was always neutral. These emotion-neutral combinations were chosen to reduce cross-contamination between positive and negative sounds within a given sequence. In pure-valence lists, both emotional event boundaries had the same valence. Based on the two event boundary positions (after the $8^{\text {th }}$ and $16^{\text {th }}$ item in a sequence), the lists were broken down as follows: 8 pure lists total (three positive-positive, three negative-negative, two neutral-neutral) and 8 mixed lists (two neutral-positive, two positive-neutral, two negative-neutral and two neutral-negative). The order of the different event boundary list types was randomized across participants, and the trial-unique naturalistic audio-clips were pseudorandomized across event boundary positions and across lists.

Delay distractor task. To reduce potential recency effects in memory, participants performed a 45-s arrow distracter task after studying each list. Participants viewed a rapid stream of either left-facing $(<)$ or right-facing $(>)$ arrow symbols. The arrows appeared in the middle of the screen for $0.5 \mathrm{~s}$ and were separated by a $0.5-\mathrm{s}$ fixation cross. Participants simply had to indicate the direction of each arrow via button press.

Temporal memory tests. Following the distracter task, participants viewed pairs of items from the prior list and had to provide a temporal distance rating and judge their correct order. First, participants rated the subjective temporal distance between two items using the following response options: 'very close', 'close', 'far' or 'very far' apart in the prior sequence (Figure 1). Second, participants indicated which of the two probe items had appeared first (temporal order memory; Figure 1). Crucially, each item pair had always been presented with three intervening items during encoding, and were thereby always encountered the same objective distance apart. Each tested item only appeared once during the temporal memory tests. To examine how emotional sounds influence temporal memory, we tested memory for two types of item pairs: (1) items that had appeared within the same auditory event and (2) items that had spanned an intervening positive, negative, or neutral audio-clip.

The structure of the event sequences as well as the specific item positions that were tested are displayed in Supplementary Figure 1. Of note, there were two item pairs spanning each naturalistic audio-clip (event boundary) and two same-context item pairs per auditory event. The specific positions of these pairs were spread out in time, enabling us to examine temporally dynamic effects of emotion on temporal memory. That is, determine whether temporal memory differed according to an item pair's proximity to a preceding emotional event boundary. Because we were interested in the discrete (e.g., boundary) and forward carryover 
effects of emotion on memory, data analyses did not include same-context item pairs from the first event of each list (i.e., the 8 items encountered before the first naturalistic audio-clip). This resulted in a total of 64 same-context pairs and 64 boundary-spanning pairs ( 4 of each type per list).

Emotional sound ratings. After the entire event boundary experiment was completed, participants listened to all of the emotional sounds again and provided separate ratings for arousal (from $1=$ "not at all" to $7=$ "extremely"), valence (from 1 = "very unpleasant" to 7 = "very pleasant"), and ambiguity (from 1 = "very easy to discriminate" to 7 = "very hard to discriminate"). Ambiguity ratings were collected as a covariate to account for variability in whether participants had trouble deciding whether a sound was positive, negative, or neutral. The three sound rating scales are depicted in the rightmost panel of Figure 1.

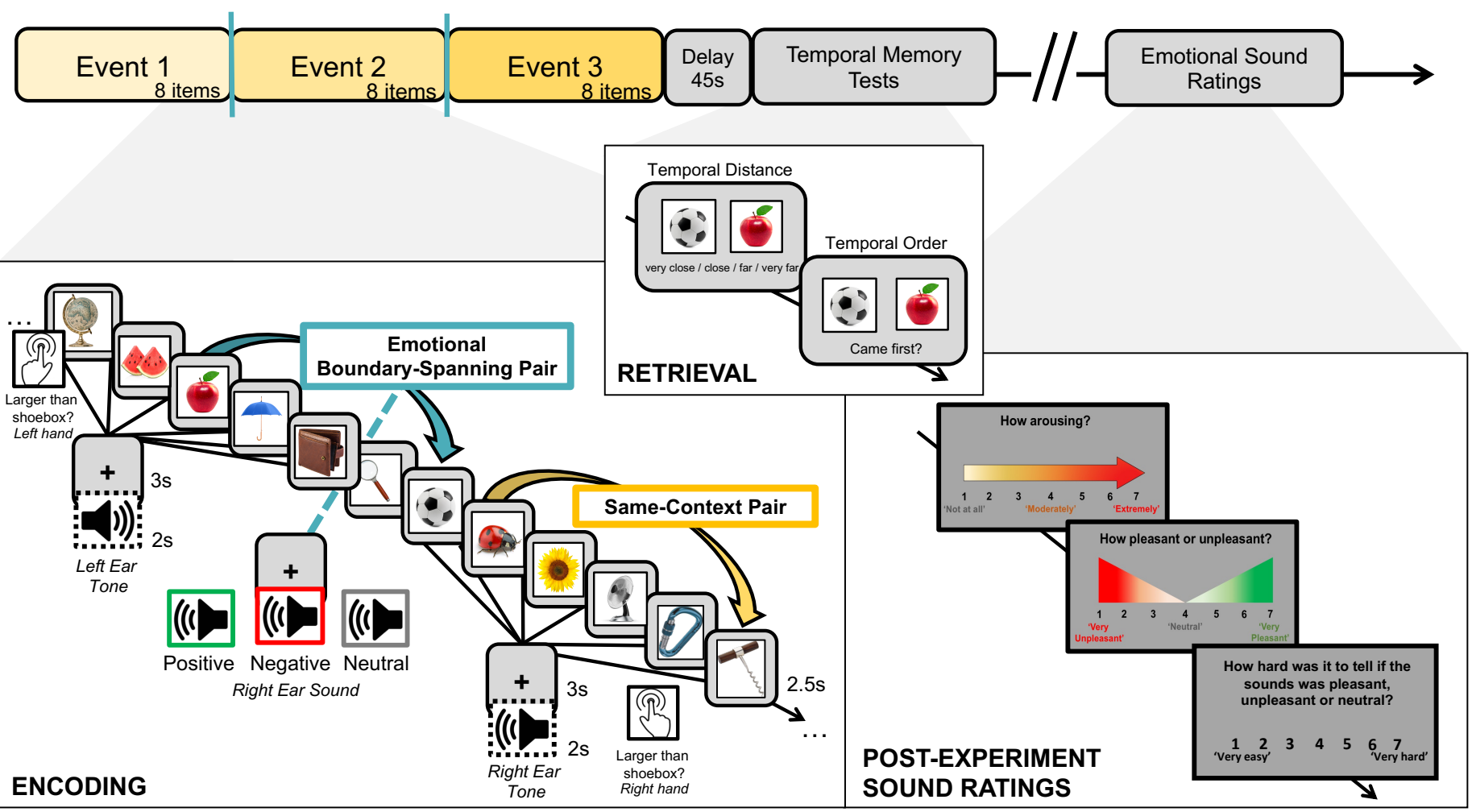

Figure 1. Schematic of the emotional auditory event boundary paradigm. Participants studied lists of 24 everyday objects and had to indicate whether each item would fit in a shoebox. The surrounding auditory context was manipulated by playing a pure tone in either participants' left or right ear prior to viewing each image. The laterality of the tone also cued which hand participants should use to make their object size judgements. After viewing 8 successive items, participants heard an emotional negative (e.g., scream), neutral (e.g., clock ticking), or positive (e.g., birds chirping) audio-clip in their other ear. Participants then heard the pure tone again in that same (new) ear prior to the next 7 items. They then heard a second, new naturalistic audio-clip in their original ear. The two naturalistic audio-clips served as event boundaries that divided each sequence into three discrete auditory events. After a short arrow distractor task, participants performed two different temporal memory tests. First, they had to rate the temporal distance between a pair of items from the prior sequence, ranging from 'very close' to 'very far' apart in the event sequence. Second, they had to indicate which of the two presented items had appeared first in the prior event sequence. The key memory comparison was between to-be-tested pairs that had spanned an emotional sound ('boundary-spanning pair'; blue) during encoding or were encountered within the same auditory event ('same-context pair'; gold). Following the event 
boundary task, participants re-listened to all of the naturalistic audio-clips from the experiment and provided subjective ratings of arousal, valence, and ambiguity.

Linear mixed modeling analyses between emotional arousal, valence, and temporal memory. To test our key hypotheses that emotional arousal and valence influence the temporal structure of memory, we performed generalized linear mixed modeling analyses using the glme4 packages in $\mathrm{R}$ (http://cran.rproject.org/web/packages/lme4/). Trial-level subjective ratings for the arousal, valence, and ambiguity for each emotional event boundary sound were mean-centered and entered into the model as fixed-effect predictors. Boundary-spanning item pairs were assigned the subjective ratings of their intervening emotional boundary sounds. Same-context item pairs were assigned the subjective ratings of the emotional boundary sounds that immediately preceded them. This enabled us to examine the proactive, or carryover, effects of emotion on encoding same-context pairs in the subsequent event. Participant was specified as a random effect, enabling us to account for individual differences in emotion-memory relationships.

To determine if emotional boundaries had different effects on temporal binding for boundary-spanning pairs compared with same-context pairs, Condition was modeled as a fixed-effect predictor $(1=$ boundaryspanning pair; -1 = same-context pair). We also included two-way interaction terms between subjective arousal and valence ratings, both of their interactions with Condition, as well as a three-way arousal-by-condition-byvalence interaction term. Temporal order memory accuracy for the item pairs was coded as a binary variable (1 = correct, 0 = wrong) and modeled as the dependent measure.

Random effects for List Type (e.g., positive-positive, neutral-positive etc.) and Event Number (event 2 or event 3) were entered as covariates in each model to account for potential differences in the time-course of arousal/valence effects and their potential interactions within a given list. For all of the analyses, the statistical significance of the regression models was determined using model comparisons, which resulted in $\chi^{2}$ values and corresponding $p$ values.

To disentangle the effects of arousal and valence on temporal memory, we also performed simple slopes analyses. Here, we examined whether the linear relationship between arousal ratings temporal order memory differed at low, moderate, and high levels of emotional valence. Fitted regression lines were plotted at low (-1 SD; negative) and moderate (average; neutral) and high (+1 SD; positive) levels of emotional sound valence ratings using estimates from the generalized linear mixed model.

\section{$\underline{\text { Results }}$}

Encoding Response Times. During encoding, we compared response times of the size judgements between boundary items and same-context items (Figure 1). Participants were slower to judge items appearing just after a boundary sound (i.e., naturalistic audio-clip; $M=1270 \mathrm{~ms}, \mathrm{SD}=220 \mathrm{~ms}$ ) than objects appearing within the same auditory context (i.e., repeated pure tone; $M=1124 \mathrm{~ms}, \mathrm{SD}=162 \mathrm{~ms} ; \mathrm{t}(19)=5.78, \mathrm{p}<.001$ ). Triallevel mixed modeling analyses of the event boundary trials revealed no significant main or interaction effects of the naturalistic sounds' valence or arousal on these response times (all p's > .05). 
Trial-level effects of emotional event boundaries on temporal order memory. To test our key hypothesis that emotional context modulates the temporal structure of memory, we performed generalized linear mixed modeling analyses using participants' trial-level subjective arousal, valence, and ambiguity ratings for each emotional sound. We found that temporal order memory was significantly better for same-context item pairs compared to boundary-spanning item pairs $\left(\chi^{2}(1)=13.93, p=.00019\right)$. This result replicates longstanding work on event cognition and supports the notion that temporal memory integration is supported by context stability while memory separation is driven by context shifts (Clewett \& Davachi, 2017; Clewett et al., 2019).

Critically, we also identified a significant arousal-by-condition interaction effect on temporal memory. As shown in Figure 2, highly arousing event boundaries were associated with: (1) worse temporal order memory for boundary-spanning pairs, and (2) better temporal order memory for same-context pairs from the next auditory event $\left(\chi^{2}(1)=7.17, p=.0074\right)$. To determine which factors drove this arousal-by-memory interaction effect on memory, we performed separate correlation analyses for each condition. We found that higher arousal ratings for boundary sounds were significantly correlated with better temporal order memory for samecontext item pairs encountered within the next auditory event $\left(\chi^{2}(1)=4.71, p=.030\right)$. By contrast, we observed a trend towards memory impairment in the relationship between arousal and memory for boundary-spanning pairs, with higher arousal ratings for boundary sounds relating to worse temporal order memory $\left(\chi^{2}(1)=1.74, p\right.$ $=$.19). The full model results with both conditions also revealed a marginally significant three-way interaction between arousal, valence, and condition on temporal memory $\left(\chi^{2}(1)=3.59, p=.058\right)$. Interestingly, we also found that sounds that were later rated as more ambiguous in valence were related to worse temporal order memory irrespective of condition $\left(\chi^{2}(1)=5.45, p=.02\right)$. There was no main effect of valence or valence-byarousal interaction effect on temporal order memory (all p's > .05). 

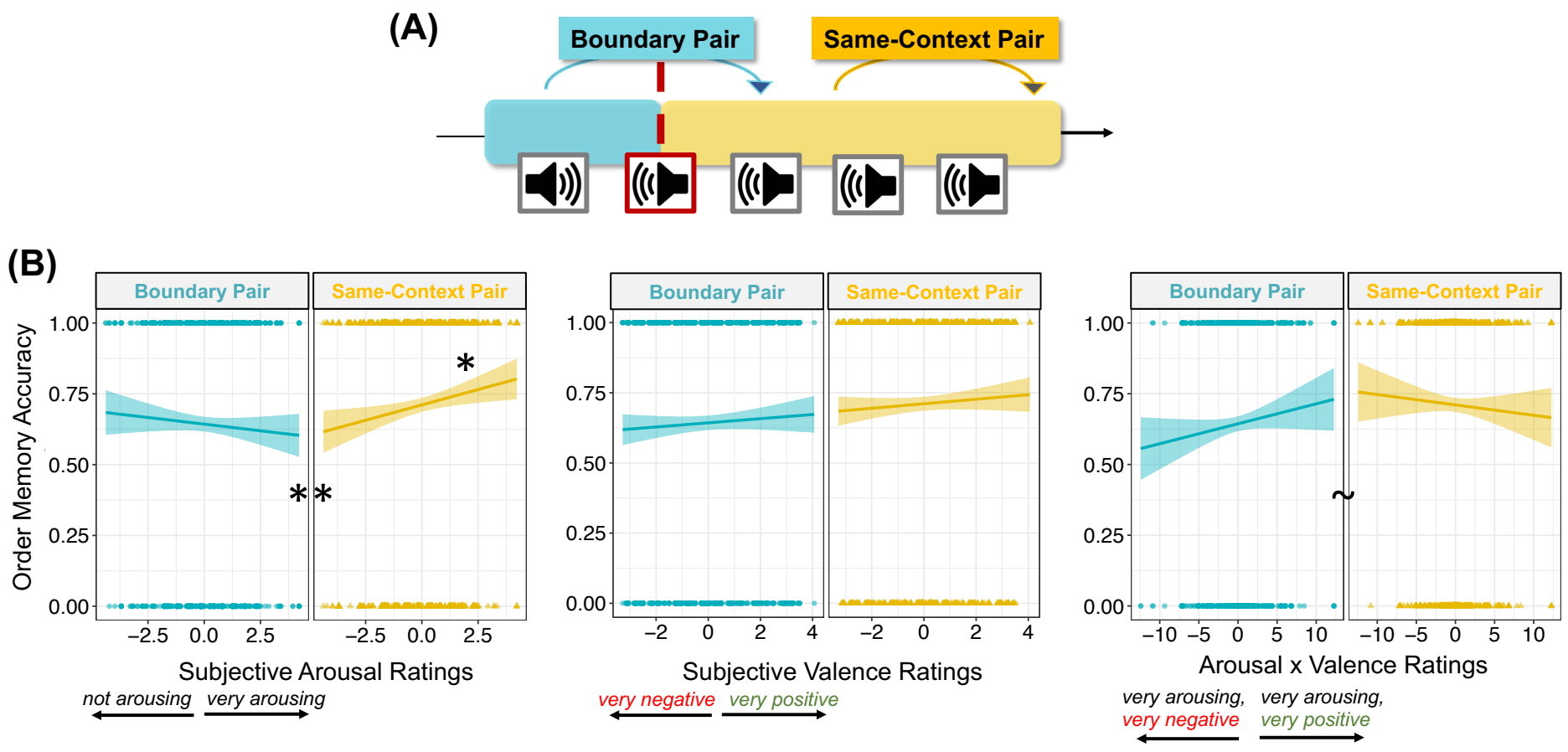

Figure 2. Generalized linear mixed modeling analyses of the relationship between subjective emotional ratings and temporal order memory. (A) Schematic of the two types of item pairs that were tested for temporal order memory after sequence encoding. The direction of the audio icons represents the ear that participants heard the sounds in. Gray-bordered icons represent pure tones, whereas the red-framed icon and red dashed line represent the emotional sound, or event boundary. Some item pairs spanned an intervening emotional sound ('boundary-spanning pair'; blue). The other item pairs were encountered within the same auditory context. That is, they were preceded by a pure tone ('same-context pair'; gold). (B) Generalized linear mixed modeling results between trial-level temporal order memory and emotional sound ratings for arousal (left panel), valence (middle panel), and their interaction (right panel). $\sim p<.10 ;{ }^{*} p<.05 ;{ }^{* *} p<.01$.

Valence-specific effects of arousing event boundaries on temporal order memory. Next, we performed a simple slopes analysis to dissociate the effects of arousal on memory at different levels of pleasantness, or valence. We adopted this approach, because it allows us to disentangle arousal-by-valence interactions that may otherwise be obscured by treating valence as a continuous variable. These analyses were performed separately for the two conditions (Figure 3).

For same-context pairs, we found that emotional event boundary sounds rated as arousing and more negative led to better temporal order memory (valence rating -1 SD below the mean; $z=2.53, p=.01$ ). That is, naturalistic sounds that were perceived as highly negative and arousing led to better temporal binding between item pairs that were encountered several seconds later. A similar arousal-memory correlation was observed for sounds rated as neutral (mean valence rating; $z=2.25, p=.02$; Figure $3 B$ ). By contrast, there was no significant association between arousal and temporal order memory when the preceding emotional event boundary sounds were rated as more positive (valence rating +1 SD above the mean; $z=0.63, p=.53$ ).

For boundary-spanning pairs, we observed an event segmentation pattern that was consistent with our prediction that negative, arousing boundaries would impair temporal memory. Emotional sounds that were rated as more negative showed a marginally significant negative association between arousal and temporal order memory (valence rating -1 SD below the mean; $z=-1.91, p=.06$ ). There were no significant 
associations between emotional arousal and temporal memory for sounds that were rated as neutral (mean valence rating; $z=-1.41 ; p=.16$ ) or more positive (valence rating +1 SD above the mean; $z=0.94, p=.94$ ).

Taken together, these findings suggest that the divergent effects of emotionally arousing event boundaries on temporal order memory emerge under increasingly aversive contexts. On the one hand, negative and arousing emotional moments tend to segment adjacent experiences in memory. On the other hand, negative and arousing-inducing stimuli promote temporal binding between ensuing information. These results underscore the temporally dynamic and complex effects of arousal on the temporal integration of negative memories, with associative binding differing according to whether an arousing context shift precedes or intervenes between the encoding of sequential information.

(A)

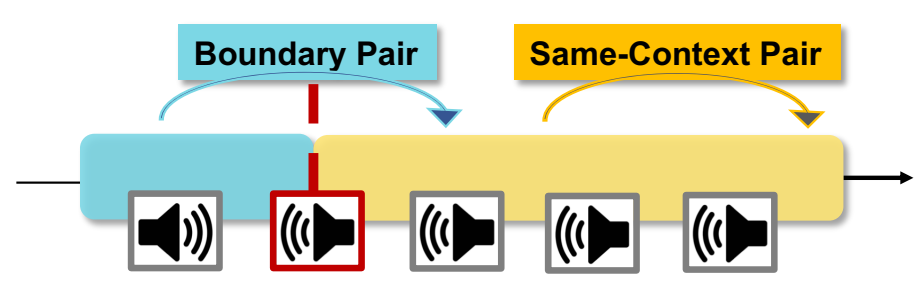

(B)

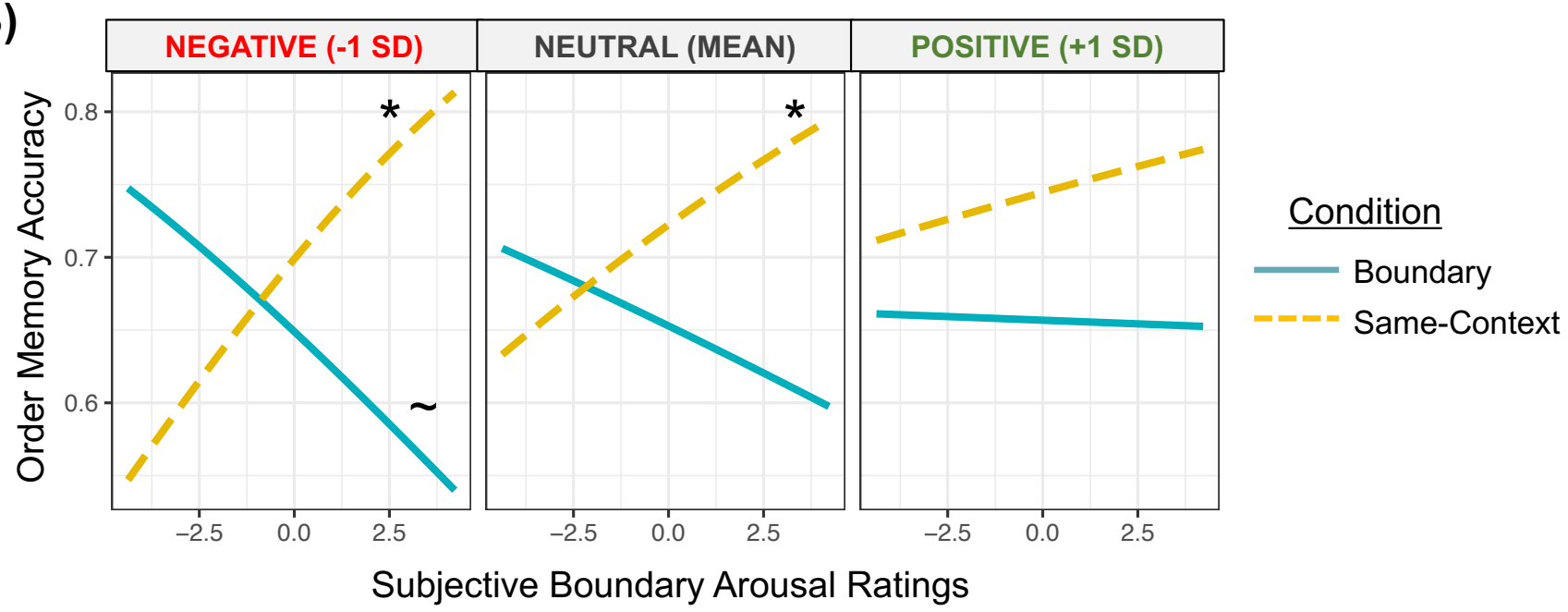

Figure 3. Temporal order memory accuracy as a function condition and the arousal and valence of emotional event boundaries. (A) Schematic of the two types of item pairs that were tested for temporal order memory after sequence encoding. The direction of the audio icons represents the ear in which participants heard the sound in each trial. Gray-bordered icons represent pure tones, whereas the red-framed icon and red dashed line represent the naturalistic sounds, or event boundaries. Some item pairs spanned an intervening emotional sound ('boundary-spanning pair'; blue and solid lines). Other item pairs were encountered within the same auditory context ('same-context pair'; gold and dashed lines). Same-context item pairs were assigned the same arousal, valence, and ambiguity ratings as the preceding naturalistic audio-clip, or event boundary. (B) Generalized linear mixed modeling results are shown separately for boundary-spanning pairs and samecontext pairs. The slopes of the lines represent the relationship between subjective arousal ratings and temporal order memory accuracy. These arousal-memory correlations are plotted separately for emotional sounds that were rated as more negative (-1 SD below the mean valence), neutral (mean valence), and more positive (+1 SD above the mean valence). $\sim p<.10 ;{ }^{*} p<.05$. 
Time-dependent effects of arousing event boundaries on same-context temporal binding. The results thus far suggest that the modulatory effects of an arousing sound linger in time to influence temporal binding processes for subsequent items. Next, we explored whether the strength of these arousal effects diminished or increased over time. Here, we used another simple slopes analysis to query whether arousal effects were stronger for same-context item pairs encountered earlier (i.e., immediately following the boundary) compared to later in the subsequent auditory event (Figure 4A). The same simple slopes analysis was conducted as before, except this time we modeled the Pair Position (early, late) of same-context item pairs as a predictor of temporal order accuracy (see Supplementary Figure 1 for an illustration of which pairs were examined).

We found a significant positive association between temporal order memory and boundary-related arousal for item pairs that were encountered just after boundaries ('early pairs') and were rated as being more negative $(z=2.62, p=.01)$ or more neutral $(z=2.27, p=.02$; Figure 4B). Thus, the arousal induced by negative event boundaries had a strong and relatively immediate influence over temporal memory binding. There was no significant association between arousal and temporal memory on trials where the preceding event boundary sounds were rated as having more positive valence (mean valence rating $+1 S D ; p=.56$ ). When instead examining same-context item pairs encountered farther into the ensuing event ('late pairs'), we did not find any significant arousal-memory correlations ( $p$ 's > .38). This finding suggests that, in aversive contexts, arousing emotional boundaries benefit temporal memory integration early on in the next event but this benefit gradually diminishes as that event unfolds.

(A)

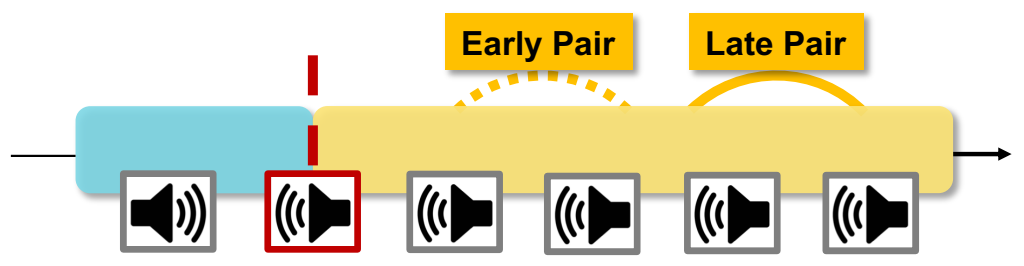

(B)

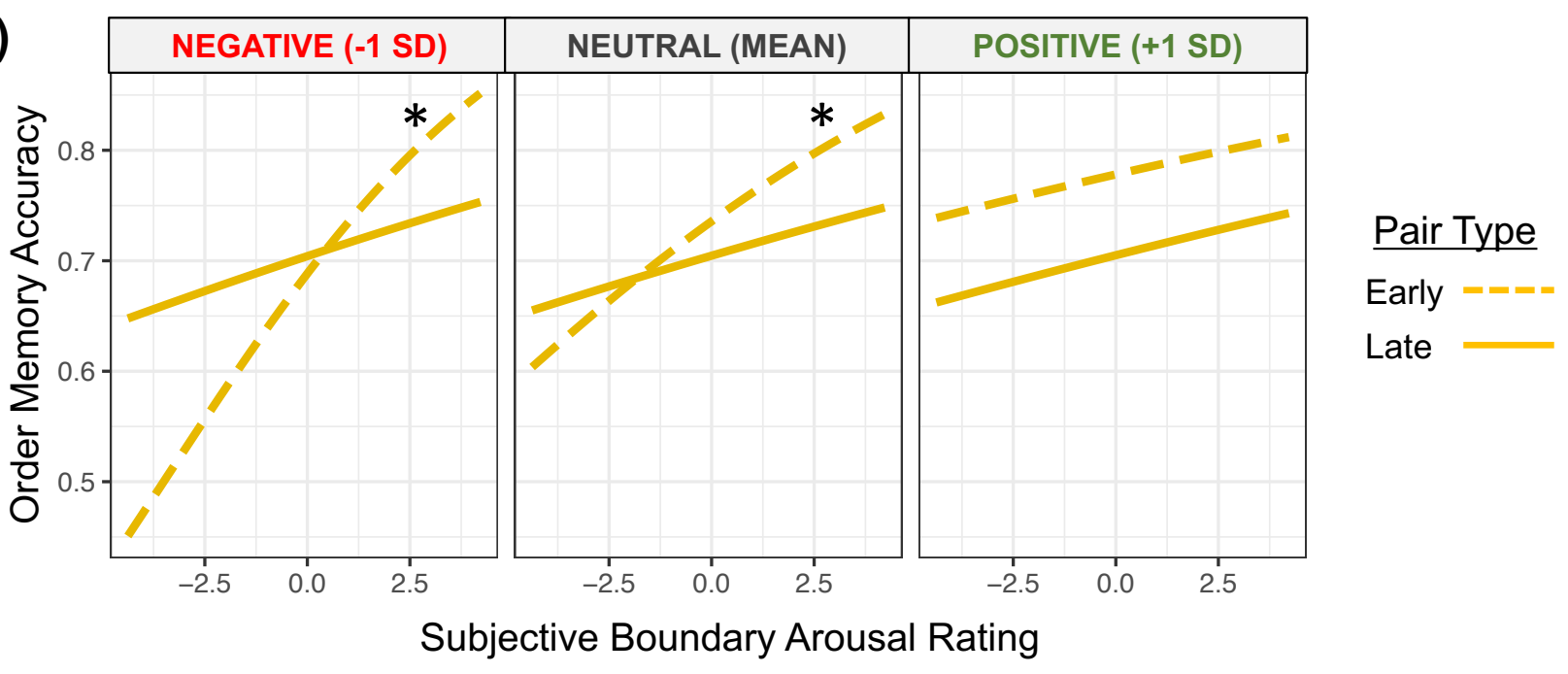

Figure 4. Temporal order memory accuracy as a function of same-context pair positions as well as the arousal and valence of the preceding emotional event boundary sounds. (A) Schematic of the two types 
of same-context item pairs that were tested for temporal order memory after sequence encoding. For half of the same-context item pairs, the first item in a to-be-tested pair was encountered just after an event boundary (position 1 in an 8-item event; Early Pair; dashed gold line). For the other half of same-context item pairs, the first item in a to-be-tested pair was encountered later on in the event (position 3 in an 8-item event; Late Pair; solid gold line). (B) Generalized linear mixed modeling results are shown separately for early and late samecontext pairs. The slopes of the lines represent the relationship between subjective arousal ratings and temporal order memory accuracy. These arousal-memory correlations are plotted separately for emotional sounds that were rated as negative (-1 SD below mean valence), neutral (mean valence), or positive (+1 SD above mean valence). ${ }^{*} \mathrm{p}<.05$.

Emotional Sound Ratings. As a manipulation check, participants rated the emotional boundary sounds as significantly differing in valence, $F(2,18)=107.58, p<.001, \eta^{2}=0.92$, arousal, $F(2,18)=31.97, p<.001, \eta^{2}=$ 0.78 , and ambiguity, $F(2,18)=25.33, p<.001, \eta^{2}=0.74$. Negative sounds were rated as being significantly more negative $(M=2.00, S D=0.70)$ than neutral sounds $(M=3.54, S D=0.45 ; t(19)=-12.64, p<.001)$, and positive sounds were rated as being significantly more positive $(M=4.81, S D=0.50)$ than neutral sounds $(t(19)=9.89, p<.001)$. Negative sounds $(M=4.70, S D=1.63)$ and positive sounds $(M=3.87, S D=1.27)$ were both rated as being significantly more arousing than neutral sounds $(M=3.02, S D=1.23$; negative: $t(19)$ $=7.40, \mathrm{p}<.001$; positive: $\mathrm{t}(19)=4.71, \mathrm{p}<.001$. Additionally, negative sounds were rated as being significantly more arousing than positive sounds $(\mathrm{t}(19)=3.67, \mathrm{p}=.002)$. Finally, the valence of neutral sounds $(\mathrm{M}=2.95$, $S D=0.97)$ was rated as being significantly more ambiguous than positive $(M=2.33, S D=0.83 ; t(19)=3.26, p$ $=.004)$ and negative sounds $(\mathrm{M}=1.93, \mathrm{SD}=0.67 ; \mathrm{t}(19)=7.04, \mathrm{p}<.001)$, and positive sounds were rated as being more ambiguous than negative sounds $(\mathrm{t}(19)=4.21, \mathrm{p}<.001)$.

\section{Discussion}

Moments of emotional reactivity punctuate everyday life and lead to the formation of lasting memories. Yet, while it is commonplace to refer these memories as emotional 'events', little empirical work has examined if and how emotion organizes continuous experiences into discrete and temporally-coherent episodes. To fill this knowledge gap, we examined how embedding emotional stimuli within otherwise neutral and continuous item sequences leads to changes in temporal order memory, a behavioral index of event formation. We also used peripheral auditory stimuli to manipulate encoding structure within these sequences, thereby creating the temporal stability and changes in contextual information that appear necessary for inducing event segmentation in perception and memory (Clewett et al., 2019; Siefke et al., 2019). Our results revealed that discrete emotional moments, such as the sound of screams or a gunshot, exert differential effects on temporal binding processes across time. Specifically, negative and arousing event boundaries tended to lead to transient impairments in temporal encoding processes, but then quickly rebounded to facilitate temporal binding processes for subsequent neutral memoranda. We interpret these differential effects of emotional arousal as reflecting enhanced integration of emotionally-relevant episodes, which may play a central role in the development and persistence of negative memories.

In addition, we found valence-specific effects of emotional arousal on temporal order memory, with negatively valenced event boundaries leading to the most robust memory segmentation and subsequent 
integration effects. Prior work demonstrates that negative affect tends to narrow the scope of attention and cognitive processing, whereas positive affect broadens them (Gable \& Harmon-Jones, 2010). In a related manner, it is well documented that negative stimuli typically disrupt the coherence of episodic memory, leading to widespread impairments in source memory for nearby neutral items (Bisby et al., 2018; Mather, 2007). By contrast, positive emotions have been shown to enhance associative memory (Madan et al., 2019), consistent with the idea that positive affect helps integrate complex elements of experience into multi-faceted mental representations (Clewett \& Murty, 2019; Fredrickson \& Branigan, 2005). Our findings build on prior research and align with recent work showing that after individuals navigate through a highly arousing and scary haunted house, they exhibit better memory for perceptual details from that experience but reduced memory for the time and temporal order compared to when they navigate through a neutral space (Reisman et al., 2021). While those findings revealed how sustained arousal and stressful states affect temporal and perceptual memory, our data suggest that punctate moments of emotional arousal have both discrete and sustained effects on memory that reflect the structure on contextually-distinct events.

Interestingly, encouraging individuals to be more item-focused during encoding has also been shown to disrupt temporal order memory (DuBrow \& Davachi, 2013), thereby mimicking the effects of negative emotion and arousal on cognitive narrowing processes. Increasing evidence also indicates that boundaries enhance attention and processing of concurrently presented item representations at the expense of binding items together across those context shifts (Heusser et al., 2018). Our findings suggest that injecting arousing boundaries with negative valence may amplify cognitive- and attention-narrowing effects even further, leading to greater memory separation between emotional and temporally adjacent neutral events. It is important to note that participants rated the negative sounds as being significantly more arousing than positive sounds, so we cannot fully verify whether our results are specifically driven by arousal effects under negative valence. For example, it is possible that positive sounds would have elicited similar effects on temporal memory if their arousal levels were comparable the arousal induced by the negative sounds. This idea is consistent with proposals that motivational intensity, a homologue of arousal, may be the driving force behind the cognitive narrowing effects that are typically observed and ascribed to negative emotions (Harmon-Jones et al., 2012).

We also found that emotion's forward carryover effects on temporal order memory may gradually diminish. Specifically, negative and arousing event boundaries selectively enhanced temporal order memory for item pairs that were encountered toward the beginning of the ensuing event but not later on. It is notable that the first item in these early, post-boundary pairs also included the "boundary" item; that is, the first object appearing after an emotional sound. Prior work shows that item and item-source memory tend to be enhanced for items that co-occur with a context shift (Clewett et al., 2020; Heusser et al., 2018; Siefke et al., 2019; Swallow et al., 2009). This raises the possibility that arousing, negative event boundaries may serve to anchor boundary representations in long-term memory, which might in turn enable individuals to use item strengthbased retrieval strategies to discriminate the primacy of those items (DuBrow \& Davachi, 2017).

Our findings indicate that negative emotion and arousal may have additive effects on binding experiences together into contextually-meaningful events. An interesting open question concerns the 
neuromechanisms that promote this hyper-integration of emotional episodic memories. Arousal-related activation of the noradrenergic system plays an essential role in enhancing processing of emotional information (Markovic et al., 2014; Mather et al., 2015; McGaugh, 2013; Strange et al., 2003). The noradrenergic system has also been shown to enhance the selectivity of perception and memory under arousal, potentially leading to trade-offs between item memory and memory for peripheral contextual information (Mather et al., 2015). Accumulated evidence also shows that the noradrenergic system modulates memory processes in the hippocampus, a region that is critical for supporting representations of context and integrating memories of time and sequential events (Clewett et al., 2019; Harley, 2007; Ranganath \& Hsieh, 2016; Sara, 2009; Squire, 1992). Interestingly, theories of noradrenergic system function and the neural processes that facilitate event segmentation also share considerable conceptual overlap. The release of NE is thought to facilitate a "network reset" that reorganizes functional brain networks to prioritize and process salient information during a shift in environmental contingencies (Bouret \& Sara, 2005). These shifts could be construed as event boundaries, which have been theorized to trigger a global neural signal that rapidly updates mental representations of the current context (Zacks et al., 2007). Given its widespread projections to most of the brain (Sara, 2009), the noradrenergic system is ideally positioned to transmit such a reset signal, while also influencing temporal encoding processes in the hippocampus.

The current findings lay important new groundwork for understanding how dynamic emotional arousal states provide a strong internal context for binding sequential representations in memory. An important open question is whether these memory-structuring effects also relate to the superior memorability of emotional experiences compared to neutral ones. Our findings suggest that contexts that unfold after an arousing and salient event boundary are more readily tagged as motivationally relevant, making an otherwise mundane and neutral experience more memorable. Indeed, there are some indications that event segmentation processes also lead to greater memory accuracy later on. For example, inserting event boundaries into a dynamic experience has been shown to boost free recall even up to one month later (Flores et al., 2017). For more stressful and highly arousing situations, however, this story may be more complex. Behavioral work shows that higher rates of event segmentation during stress-inducing movies instead relates to worse item recognition memory for those films (Sherrill et al., 2019). This negative association between event segmentation performance and long-term memory was also related to greater state anxiety across participants. Further research is needed to better understand how arousal and anxiety influence event perception and its complex relationship with long-term memory.

Insofar as event segmentation does lead to better long-term recall, our findings suggest that parsing and binding of emotionally arousing contexts may contribute to the persistence of aversive memories. This idea is supported by evidence that transitions between fear learning and extinction, which could be construed of as an event boundary, enhance recognition memory for items appearing just prior to those transitions (Dunsmoor et al., 2018). However, this result only speaks to how a coarse transition between two types of emotional learning influences memory for preceding item representations. By using temporal memory indices of event structure as well as a large number of item sequences/trials, we demonstrate that emotional contexts 
indeed shape the temporal structure of memory as an experience unfolds. This process of arousal-enhanced event segmentation may bind otherwise neutral representations to an underlying emotional context, which could make items strong retrieval cues for other emotionally-relevant information encountered in that event (Talmi et al., 2019). At the same time, computational models suggest that such enhancements might also disrupt the scaffolding effects of other forms of context, such as time, on memory encoding and retrieval (Talmi et al., 2019).

Several limitations in our study warrant consideration. While we had adequate statistical power to detect emotional boundary effects on temporal order memory, our study had a relatively modest sample size. Due to unforeseen disruptions in data collection during the COVID-19 pandemic, we lacked sufficient statistical power to examine how emotional boundaries affect subjective aspects of temporal memory (i.e., temporal distance ratings). Examining these effects would help shed additional light on how emotional states shape episodic memory organization as well as internal representations of time. To avoid creating inadvertent 'event boundaries' during encoding, we also had participants re-listen to the emotional sounds and provide subjective arousal, valence, and ambiguity ratings after the entire task was completed. However, acquiring postexperiment ratings prevented us from measuring emotional responses as they first occurred during sequence encoding. Future studies could use objective measures of physiological arousal, such as pupillometry or skin conductance, to evaluate initial emotional responses without disrupting attention during the sequence encoding task. Collecting continuous pupil measures would be especially useful for tracking the time-course of fluctuating arousal states across the course of encoding. Of relevance to our current findings, it has been shown that the temporal stability of arousal states, as indexed by variability in pupil diameter over time, relates to temporal memory integration (Clewett et al., 2020). Viewed through this lens, the current findings may reflect aversive boundaries eliciting an elevated yet stable state of arousal that transiently benefits temporal memory integration.

Unexpectedly, we also found that sounds that were perceived as being more ambiguous in valence were associated with worse temporal order memory irrespective of condition. We collected these ratings to control for variability in the emotional complexity of naturalistic sounds. However, we had no a priori predictions about how these ratings would relate to temporal memory. We speculate, however, that sounds that are more obscure or ambiguous might have distracted participants from the task at-hand and perhaps occupied attentional resources that were necessary for holding sequential items in working memory. An interesting direction for future research will be to dissociate how different sensory and semantic features of complex sounds impact dynamic attentional and temporal encoding processes.

Characterizing how shifting emotional states organize memories is essential for understanding how prior experiences can guide both adaptive or maladaptive behaviors. Under normal circumstances, the process of event segmentation may adaptively chunk experiences to facilitate the long-term storage and retrieval of everyday memories (Flores et al., 2017; Gold et al., 2017; McGatlin et al., 2019). However, the robust memorystructuring effects of emotional arousal may become maladaptive if this makes aversive memories more resistant to updating or forgetting. For instance, failures to encode or remember the temporal order of highly 
emotional events is a hallmark feature of post-traumatic stress disorder (Hackmann et al., 2004; van der Kolk \& Fisler, 1995). It is thought that individuals with post-traumatic stress disorder (PTSD) preferentially process perceptual information at the expense of surrounding contextual details, including the spatial or temporal context (Ehlers, 2006; Ehlers \& Clark, 2000; Ehlers et al., 2002). Again, this bears a striking resemblance to the memory trade-off effects have been shown to occur during neutral context shifts, with boundaries prioritizing the processing of novel incoming information at the expense of maintaining the encoding of temporal associations (Heusser et al., 2018). If successful temporal binding is a strong predictor of long-term memory, it may be the case that diminishing the arousal of emotional event boundaries helps deconstruct and reduce the durability of traumatic episodes. The current findings open up several exciting avenues for identifying cognitive and neural factors that facilitate the persistent and potentially debilitating effects of emotional memories in healthy individuals and in individuals with PTSD. This knowledge can in turn be leveraged to improve therapies that treat episodic memory dysfunction in various arousal-related disorders, including PTSD, perhaps by restoring links between individual emotional details and their original temporal context. 


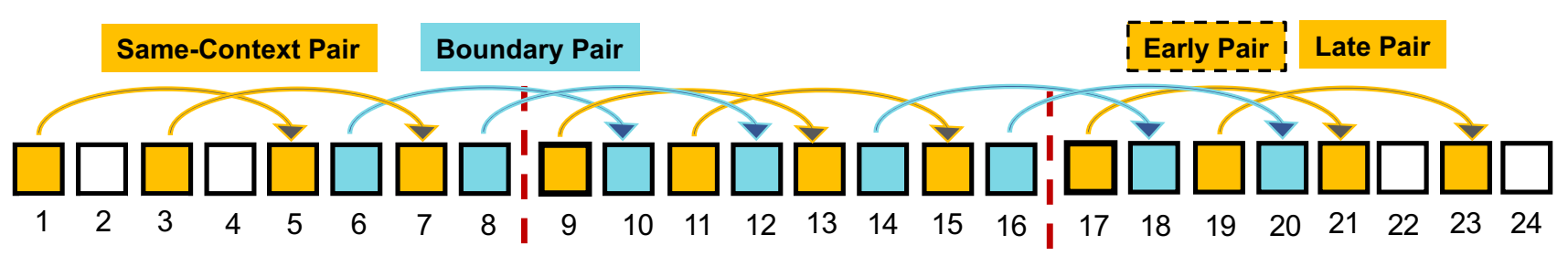

Supplementary Figure 1. Schematic of the item pair positions within a sequence. During encoding, some of the to-be-tested item pairs spanned an intervening naturalistic audio-clip that could be emotionally negative, neutral, or positive ('boundary-spanning'; blue). Other to-be-tested item pairs were encountered within a shared auditory context ('same-context'; gold), indexed by repetitions of the same pure tone before each item. There were also two types of same-context item pairs that were tested later on. Half of these pairs included an item immediately following an event boundary (dashed box), whereas the other half were encountered slightly farther into the event. While only two of these pairs are labeled in the figure, these pairs were present in each of the three auditory events. 


\section{References}

Anderson, A. K., Wais, P. E., \& Gabrieli, J. D. E. (2006, Jan). Emotion enhances remembrance of neutral events past. Proceedings of the National Academy of Sciences of the United States of America, 103(5), 1599-1604. https://doi.org/10.1073/pnas.0506308103

Barnacle, G. E., Tsivilis, D., Schaefer, A., \& Talmi, D. (2018, Apr). Local context influences memory for emotional stimuli but not electrophysiological markers of emotion-dependent attention.

Psychophysiology, 55(4). https://doi.org/10.1111/psyp.13014

Bisby, J. A., \& Burgess, N. (2014). Negative affect impairs associative memory but not item memory. Learning \& Memory, 21(1), 21-27.

Bisby, J. A., Horner, A. J., Bush, D., \& Burgess, N. (2018, Feb). Negative emotional content disrupts the coherence of episodic memories. J Exp Psychol Gen, 147(2), 243-256.

https://doi.org/10.1037/xge0000356

Bocanegra, B. R., \& Zeelenberg, R. (2009, Dec). Dissociating emotion-induced blindness and hypervision. Emotion, 9(6), 865-873. https://doi.org/10.1037/a0017749

Bouret, S., \& Sara, S. J. (2005, 11//). Network reset: a simplified overarching theory of locus coeruleus noradrenaline function. Trends in Neurosciences, 28(11), 574-582.

https://doi.org/http://dx.doi.org/10.1016/j.tins.2005.09.002

Bradley, B. P., Mogg, K., Millar, N., \& Bonham-Carter, C. (1997). Attentional biases for emotional faces. Cognition \& Emotion, 11(1), 25-42.

Bradley, M. M., \& Lang, P. J. (2007). The International Affective Digitized Sounds (2nd Edition; IADS-2): Affective ratings of sounds and instruction manual. University of Florida.

Brunec, I. K., Moscovitch, M., \& Barense, M. D. (2018). Boundaries Shape Cognitive Representations of Spaces and Events. Trends in cognitive sciences.

Brunec, I. K., Ozubko, J. D., Barense, M. D., \& Moscovitch, M. (2017). Recollection-dependent memory for event duration in large-scale spatial navigation. Learning \& Memory, 24(3), 104-114.

Cahill, L., \& McGaugh, J. L. (1998). Mechanisms of emotional arousal and lasting declarative memory. Trends in Neurosciences, 21(7), 294-299.

Clewett, D., \& Davachi, L. (2017). The ebb and flow of experience determines the temporal structure of memory. Current opinion in behavioral sciences, 17, 186-193.

Clewett, D., DuBrow, S., \& Davachi, L. (2019). Transcending time in the brain: How event memories are constructed from experience. Hippocampus, 29(3), 162-183. 
Clewett, D., Gasser, C., \& Davachi, L. (2020, Aug 11). Pupil-linked arousal signals track the temporal organization of events in memory. Nat Commun, 11(1), 4007. https://doi.org/10.1038/s41467-020$\underline{17851-9}$

Clewett, D., \& Murty, V. P. (2019, Mar-Apr). Echoes of Emotions Past: How Neuromodulators Determine What We Recollect. eNeuro, 6(2). https://doi.org/10.1523/ENEURO.0108-18.2019

Clewett, D., Sakaki, M., Nielsen, S., Petzinger, G., \& Mather, M. (2017). Noradrenergic mechanisms of arousal's bidirectional effects on episodic memory. Neurobiology of Learning and Memory, 137, 1-14.

D'Argembeau, A., \& Van der Linden, M. (2005, Dec). Influence of emotion on memory for temporal information. Emotion, 5(4), 503-507. <Go to ISI>://000234154700012

Dolan, R. J. (2002). Emotion, cognition, and behavior. Science, 298(8), 1191-1194.

Droit-Volet, S., \& Gil, S. (2009). The time-emotion paradox. Philosophical Transactions of the Royal Society of London B: Biological Sciences, 364(1525), 1943-1953.

DuBrow, S., \& Davachi, L. (2013). The influence of context boundaries on memory for the sequential order of events. Journal of Experimental Psychology: General, 142(4), 1277.

DuBrow, S., \& Davachi, L. (2014). Temporal memory is shaped by encoding stability and intervening item reactivation. Journal of Neuroscience, 34(42), 13998-14005.

DuBrow, S., \& Davachi, L. (2017). Commentary: Distinct neural mechanisms for remembering when an event occurred. Frontiers in Psychology, 8.

Dunsmoor, J. E., Kroes, M. C. W., Moscatelli, C. M., Evans, M. D., Davachi, L., \& Phelps, E. A. (2018, Apr). Event segmentation protects emotional memories from competing experiences encoded close in time. Nat Hum Behav, 2(4), 291-299. https://doi.org/10.1038/s41562-018-0317-4

Ehlers, A. (2006, Jun). More evidence for the role of persistent dissociation in PTSD. Am J Psychiatry, 163(6), 1112. https://doi.org/10.1176/ajp.2006.163.6.1112

Ehlers, A., \& Clark, D. M. (2000, Apr). A cognitive model of posttraumatic stress disorder. Behav Res Ther, 38(4), 319-345. https://doi.org/10.1016/s0005-7967(99)00123-0

Ehlers, A., Hackmann, A., Steil, R., Clohessy, S., Wenninger, K., \& Winter, H. (2002, Sep). The nature of intrusive memories after trauma: the warning signal hypothesis. Behav Res Ther, 40(9), 995-1002. https://doi.org/10.1016/s0005-7967(01)00077-8 
Ezzyat, Y., \& Davachi, L. (2014). Similarity breeds proximity: pattern similarity within and across contexts is related to later mnemonic judgments of temporal proximity. Neuron, 81(5), 1179-1189.

Faber, M., \& Gennari, S. P. (2015). In search of lost time: Reconstructing the unfolding of events from memory. Cognition, 143, 193-202.

Flores, S., Bailey, H., Eisenberg, M., \& Zacks, J. (2017). Event Segmentation Improves Event Memory up to One Month Later. Journal of experimental psychology. Learning, memory, and cognition.

Fredrickson, B. L., \& Branigan, C. (2005). Positive emotions broaden the scope of attention and thought-action repertoires. Cognition \& Emotion, 19(3), 313-332. https://doi.org/10.1080/02699930441000238

Gable, P. A., \& Harmon-Jones, E. (2008, May). Approach-motivated positive affect reduces breadth of attention. Psychological Science, 19(5), 476-482. <Go to ISI>://000255662300011

Gable, P. A., \& Harmon-Jones, E. (2010, Feb). The blues broaden, but the nasty narrows: Attentional consequences of negative affects low and high in motivational intensity. Psychological Science, 21(2), 211-215. https://doi.org/10.1177/0956797609359622

Gabrieli, J. D. E., Brewer, J. B., Desmond, J. E., \& Glover, G. H. (1997). Seperate neural bases of two fundamental memory processes in the human medial temporal lobe. Science, 276, 264-266.

Gold, D. A., Zacks, J. M., \& Flores, S. (2017). Effects of cues to event segmentation on subsequent memory. Cognitive Research: Principles and Implications, 2(1), 1.

Hackmann, A., Ehlers, A., Speckens, A., \& Clark, D. M. (2004, Jun). Characteristics and content of intrusive memories in PTSD and their changes with treatment. J Trauma Stress, 17(3), 231-240. https://doi.org/10.1023/B:JOTS.0000029266.88369.fd

Harley, C. W. (2007). Norepinephrine and the dentate gyrus. Dentate Gyrus: A Comphrehensive Guide to Structure, Function, and Clinical Implications, 163, 299-318. https://doi.org/10.1016/s0079$\underline{6123(07) 63018-0}$

Harmon-Jones, E., Gable, P. A., \& Price, T. F. (2012). The influence of affective states varying in motivational intensity on cognitive scope. Frontiers in integrative neuroscience, 6.

Heusser, A. C., Ezzyat, Y., Shiff, I., \& Davachi, L. (2018). Perceptual boundaries cause mnemonic trade-offs between local boundary processing and across-trial associative binding. Journal of Experimental Psychology: Learning, Memory, and Cognition.

Horner, A. J., Bisby, J. A., Wang, A., Bogus, K., \& Burgess, N. (2016). The role of spatial boundaries in shaping long-term event representations. Cognition, 154, 151-164. 
Huntjens, R. J., Wessel, I., Postma, A., van Wees-Cieraad, R., \& de Jong, P. J. (2015, Jul). Binding Temporal Context in Memory: Impact of Emotional Arousal as a Function of State Anxiety and State Dissociation. J Nerv Ment Dis, 203(7), 545-550. https://doi.org/10.1097/NMD.0000000000000325

Hurlemann, R., Hawellek, B., Matusch, A., Kolsch, H., Wollersen, H., Madea, B., Vogeley, K., Maier, W., \& Dolan, R. J. (2005, Jul 6). Noradrenergic modulation of emotion-induced forgetting and remembering. Journal of Neuroscience, 25(27), 6343-6349. https://doi.org/10.1523/JNEUROSCI.0228-05.2005

Johnson, L. W., \& MacKay, D. G. (2019, Mar). Relations between emotion, memory encoding, and time perception. Cogn Emot, 33(2), 185-196. https://doi.org/10.1080/02699931.2018.1435506

Kahneman, D., \& Beatty, J. (1966). Pupil diameter and load on memory. Science, 154(3756), 1583-1585.

Kensinger, E. A. (2004). Remembering emotional experiences: The contribution of valence and arousal. Reviews in the Neurosciences, 15(4), 241-251. <Go to ISI>://000224545900002

Kensinger, E. A. (2009). Remembering the details: Effects of emotion. Emotion Review, 1, 99-113. https://doi.org/10.1177/1754073908100432.

Kensinger, E. A., Garoff-Eaton, R. J., \& Schacter, D. L. (2006, Jan). Memory for specific visual details can be enhanced by negative arousing content. Journal of Memory and Language, 54(1), 99-112. https://doi.org/10.1016/j.jml.2005.05.005

Kensinger, E. A., Garoff-Eaton, R. J., \& Schacter, D. L. (2007, Nov). How negative emotion enhances the visual specificity of a memory. Journal of Cognitive Neuroscience, 19(11), 1872-1887. <Go to ISI>://000250669900012

Knight, M., \& Mather, M. (2009, Dec). Reconciling findings of emotion-induced memory enhancement and impairment of preceding items. Emotion, 9(6), 763-781. https://doi.org/10.1037/a0017281

LaBar, K. S., \& Cabeza, R. (2006, Jan). Cognitive neuroscience of emotional memory. Nat Rev Neurosci, 7(1), 54-64. https://doi.org/10.1038/nrn1825

Lake, J. I., LaBar, K. S., \& Meck, W. H. (2016). Emotional modulation of interval timing and time perception. Neuroscience \& Biobehavioral Reviews, 64, 403-420.

Liverence, B. M., \& Scholl, B. J. (2012, Jun). Discrete events as units of perceived time. J Exp Psychol Hum Percept Perform, 38(3), 549-554. https://doi.org/10.1037/a0027228

Long, N. M., Danoff, M. S., \& Kahana, M. J. (2015, Oct). Recall dynamics reveal the retrieval of emotional context. Psychon Bull Rev, 22(5), 1328-1333. https://doi.org/10.3758/s13423-014-0791-2 
Madan, C. R., Scott, S. M. E., \& Kensinger, E. A. (2019, Jun). Positive emotion enhances association-memory. Emotion, 19(4), 733-740. https://doi.org/10.1037/emo0000465

Maran, T., Sachse, P., Martini, M., Weber, B., Pinggera, J., Zugal, S., \& Furtner, M. (2017). Lost in time and space: states of high arousal disrupt implicit acquisition of spatial and sequential context information. Frontiers in Behavioral Neuroscience, 11, 206.

Markovic, J., Anderson, A. K., \& Todd, R. M. (2014). Tuning to the significant: Neural and genetic processes underlying affective enhancement of visual perception and memory. Behavioural Brain Research, 259, 229-241. http://www.sciencedirect.com/science/article/pii/S0166432813006955

Mather, M. (2007, Mar). Emotional arousal and memory binding: An object-based framework. Perspectives on Psychological Science, 2(1), 33-52. https://doi.org/10.1111/j.1745-6916.2007.00028.x

Mather, M., Clewett, D., Sakaki, M., \& Harley, C. W. (2015). Norepinephrine ignites local hot spots of neuronal excitation: how arousal amplifies selectivity in perception and memory. Behavioral and Brain Sciences, 1-100.

Mather, M., \& Sutherland, M. R. (2011). Arousal-biased competition in perception and memory. Perspectives on Psychological Science, 6, 114-133. http://pps.sagepub.com/content/6/2/114.short

McGatlin, K. C., Newberry, K. M., \& Bailey, H. R. (2019, Jan). Temporal Chunking Makes Life's Events More Memorable. Open Psychol, 1(1), 94-105. https://doi.org/10.1515/psych-2018-0007

McGaugh, J. L. (2013). Making lasting memories: Remembering the significant. Proceedings of the National Academy of Sciences, 110(Supplement 2), 10402-10407.

http://www.pnas.org.idpproxy.reading.ac.uk/content/110/Supplement 2/10402.abstract

Palombo, D. J., \& Cocquyt, C. (2020, Sep). Emotion in Context: Remembering When. Trends Cogn Sci, 24(9), 687-690. https://doi.org/10.1016/j.tics.2020.05.017

Palombo, D. J., Te, A. A., Checknita, K. J., \& Madan, C. R. (2021, Jul). Exploring the Facets of Emotional Episodic Memory: Remembering "What," "When," and "Which". Psychol Sci, 32(7), 1104-1114. https://doi.org/10.1177/0956797621991548

Pierce, B. H., \& Kensinger, E. A. (2011, Feb). Effects of emotion on associative recognition: valence and retention interval matter. Emotion, 11(1), 139-144. https://doi.org/10.1037/a0021287

Preuschoff, K., Marius't Hart, B., \& Einhäuser, W. (2011). Pupil dilation signals surprise: evidence for noradrenaline's role in decision making. Frontiers in neuroscience, 5.

Raisig, S., Welke, T., Hagendorf, H., \& van der Meer, E. (2010, Apr). I spy with my little eye: detection of temporal violations in event sequences and the pupillary response. Int J Psychophysiol, 76(1), 1-8. https://doi.org/10.1016/j.ijpsycho.2010.01.006 
Ranganath, C., \& Hsieh, L. T. (2016). The hippocampus: a special place for time. Annals of the New York Academy of Sciences, 1369(1), 93-110.

Rimmele, U., Davachi, L., \& Phelps, E. A. (2012, Aug). Memory for time and place contributes to enhanced confidence in memories for emotional events. Emotion, 12(4), 834-846. https://doi.org/10.1037/a0028003

Rouhani, N., Norman, K. A., Niv, Y., \& Bornstein, A. M. (2019). Reward prediction errors create event boundaries in memory. bioRxiv, 725440.

Sakaki, M., Fryer, K., \& Mather, M. (2014). Emotion strengthens high priority memory traces but weakens low priority memory traces. Psychological Science, 25(387-395).

http://pss.sagepub.com/content/25/2/387.abstract

Sara, S. J. (2009). The locus coeruleus and noradrenergic modulation of cognition. Nature Reviews Neuroscience, 10(3), 211-223. http://dx.doi.org/10.1038/nrn2573

Schluter, H., Hacklander, R. P., \& Bermeitinger, C. (2019, Oct). Emotional oddball: A review on memory effects. Psychon Bull Rev, 26(5), 1472-1502. https://doi.org/10.3758/s13423-019-01658-X

Schmidt, K., Patnaik, P., \& Kensinger, E. A. (2011). Emotion's influence on memory for spatial and temporal context. Cognition and Emotion, 25(2), 229-243.

Schmidt, S. R., \& Schmidt, C. R. (2016, Aug). The emotional carryover effect in memory for words. Memory, 24(7), 916-938. https://doi.org/10.1080/09658211.2015.1059859

Sherrill, A. M., Kurby, C. A., Lilly, M. M., \& Magliano, J. P. (2019, Feb). The effects of state anxiety on analogue peritraumatic encoding and event memory: introducing the stressful event segmentation paradigm. Memory, 27(2), 124-136. https://doi.org/10.1080/09658211.2018.1492619

Siefke, B. M., Smith, T. A., \& Sederberg, P. B. (2019). A context-change account of temporal distinctiveness. Memory \& cognition, 1-15.

Squire, L. R. (1992). Memory and the hippocampus: A synthesis from findings with rats, monkeys, and humans. Psychological Review, 99, 195-231.

Strange, B. A., Hurlemann, R., \& Dolan, R. J. (2003, Nov). An emotion-induced retrograde amnesia in humans is amygdala- and beta-adrenergic-dependent. Proceedings of the National Academy of Sciences of the United States of America, 100(23), 13626-13631. https://doi.org/10.1073/pnas.1635116100

Swallow, K. M., Zacks, J. M., \& Abrams, R. A. (2009). Event boundaries in perception affect memory encoding and updating. Journal of Experimental Psychology: General, 138(2), 236. 
Talmi, D., Lohnas, L. J., \& Daw, N. D. (2019, Jul). A retrieved context model of the emotional modulation of memory. Psychol Rev, 126(4), 455-485. https://doi.org/10.1037/rev0000132

van der Kolk, B. A., \& Fisler, R. (1995, Oct). Dissociation and the fragmentary nature of traumatic memories: overview and exploratory study. J Trauma Stress, 8(4), 505-525. https://doi.org/10.1007/BF02102887

Zacks, J. M., Speer, N. K., Swallow, K. M., Braver, T. S., \& Reynolds, J. R. (2007). Event perception: a mindbrain perspective. Psychological bulletin, 133(2), 273.

Zlomuzica, A., Preusser, F., Totzeck, C., Dere, E., \& Margraf, J. (2016). The impact of different emotional states on the memory for what, where and when features of specific events. Behavioural brain research, 298, 181-187. 